\title{
Treatment effects in prostate cancer
}

\author{
Andrew J Evans \\ Department of Laboratory Information Support Systems, University Health Network, Toronto General \\ Hospital, Toronto, ON, Canada
}

\begin{abstract}
Nonsurgical treatments for prostate cancer include androgen-deprivation therapy (ADT), radiation therapy (RT), ablative therapies, chemotherapy, and newly emerging immunotherapies. These approaches can be used alone or in combination depending on the clinical scenario. ADT is typically reserved for high-risk locally or systemically advanced disease that is not amenable to curative surgery. Radiation therapy can be used instead of surgery as primary therapy with curative intent for low-intermediate-risk disease as well as for control of locally advanced disease not suitable for surgery. Ablative therapies can be used as primary therapy for lowintermediate-risk disease or as salvage therapy for clinically localized disease where RT has failed. Chemotherapy and immune-based therapies are currently used for androgen-independent disease, although the indications for these approaches may well change as new data from clinical trials accrue. Pathologists should be able to recognize tissue changes associated with these treatments to provide information that will optimize patient management. This is particularly true in situations where clinical history of recent or remote nonsurgical treatment is not provided with the specimen. In the absence of this information, pathologists encountering the features described herein are encouraged to review patient records or communicate directly with clinical colleagues to determine how a given patient was treated and when.

Modern Pathology (2018) 31, S110-S121; doi:10.1038/modpathol.2017.158
\end{abstract}

In 2017, men diagnosed with prostate cancer have a wide variety of management options depending on the risk category into which their disease falls. Men with clinically low-risk prostate cancer, and some selected patients with intermediate-risk disease, frequently opt for active surveillance (AS). ${ }^{1}$ Others in the low-intermediate-risk category may choose minimally invasive ablative therapies, ${ }^{2,3}$ radiation therapy (RT), or radical prostatectomy with the intent of curing their disease. ${ }^{4}$ Men with localized or metastatic high-risk disease will typically be treated with RT with or without hormonal therapy, while patients with recurrent or metastatic, castratesensitive or -resistant disease can be offered chemotherapy. ${ }^{5-8}$ The potential of targeted therapies based on leveraging of the patient's immune system to treat the cancer is currently being actively evaluated..$^{9,10}$ Most of the aforementioned nonsurgical treatments can have marked effects on the histological appearance of tumor and non-tumor prostate tissue, which have been well described. ${ }^{11-14}$

Correspondence: Dr AJ Evans MD, PhD, FACP, FRCPC, Department of Laboratory Information Support Systems, University Health Network, Toronto General Hospital, 11-Eaton 223, 200 Elizabeth Street, Toronto, ON M5G 2C4, Canada.

E-mail: andrew.evans@uhn.ca

Received 23 July 2017; revised 24 September 2017; accepted 24 September 2017
Accurate interpretation of post-treatment prostate cancer specimens by pathologists requires awareness of the various treatment options for prostate cancer, as well as knowledge of the morphological changes associated with each treatment. In addition, pathologists also require access to clinical information such as how a given patient was treated and when. All too frequently biopsy and surgical specimens are received in the pathology laboratory with no clinical information. In such cases the pathologist must recognize treatment-related changes and obtain clinical information from an electronic patient record or through communication with clinicians. Such instances present the pathologist with the opportunity to remind clinical colleagues of the need to supply information on how a patient may have been treated as part of quality patient care.

This review will cover nonsurgical treatment modalities for prostate cancer including the following: androgen-deprivation therapy (ADT); RT; ablative therapies; chemotherapy; emerging immune therapies; as well as nutritional/herbal supplements. The reader should be able to describe effects each of the above treatments have on the morphology of prostatic adenocarcinoma, if any, and to recognize situations in which Gleason grading such specimens is not applicable. The review will also cover changes seen in benign prostatic glands and stroma, as such changes can provide important clues that a patient 

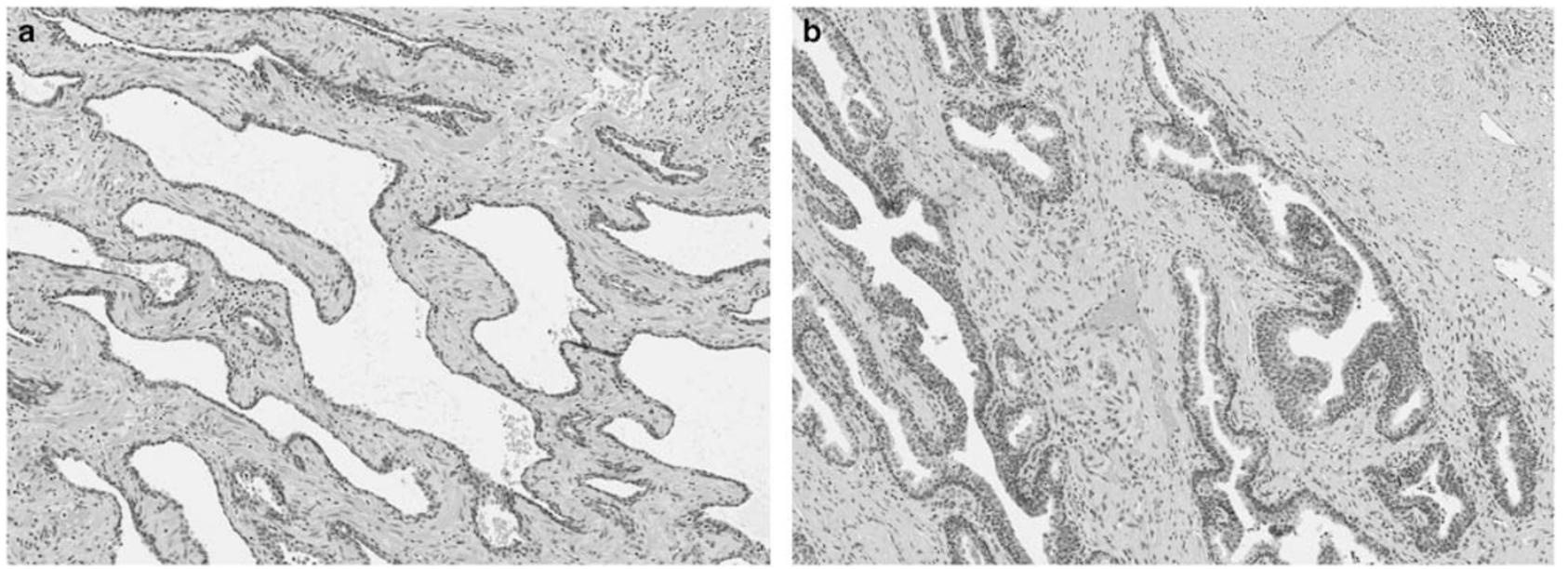

Figure 1 Typical appearance of benign prostatic glands in the setting of maximum androgen blockade. (a) Peripheral zone glands tend to show marked atrophy with prominent basal cells (hematoxylin-eosin, $\times 100$ ), while (b) transition zone glands tend to show prominent basal cell hyperplasia (hematoxylin-eosin, $\times 100$ ).

has been treated with one or more of the aforementioned modalities.

\section{Androgen-deprivation therapy}

ADT is typically used in advanced disease, most often in the setting of metastases, as a means of slowing tumor progression and alleviating symptoms. The aim is to achieve maximum androgen blockade (MAB) with castrate levels of testosterone and to maintain MAB until the inevitable emergence of castrate-resistant disease. ${ }^{10} \mathrm{ADT}$ can also be given in a neoadjuvant setting before radical prostatectomy for a variety of reasons. ${ }^{15,16}$ Limited ADT can also be used before ablative therapies such as high-intensity focused ultrasound (HIFU) to reduce prostate volume. ${ }^{17,18} \mathrm{MAB}$ can be achieved through medical or surgical castration, the latter now being used much less frequently. Medical castration involves the use of drugs that block the synthesis of testosterone or block the androgen receptor (AR). These agents can be used as monotherapy or in combination. Examples of drugs that block the synthesis of testosterone include leuteinizing hormone-releasing hormone (LHRH) agonists (eg, leuprolide and goserelin), LHRH antagonists (eg, degarelix), and cytochrome p450 inhibitors (eg, abiraterone and ketoconazole). Agents that block the AR can be steroidal (eg, cyproterone acetate) or nonsteroidal (eg, flutamide, bicalutamide, and enzalutamide). Drugs that inhibit the enzyme $5 \alpha$-reductase (5ARI; eg, finasteride and dutasteride) are considered a milder form of ADT used primarily for symptomatic benign prostatic hyperplasia (BPH), although several clinical trials exploring the efficacy of 5ARIs in the primary prevention of prostate cancer have been conducted (5ARI trials). ADT-induced changes in benign and malignant prostate tissue have been well described previously. ${ }^{11-13,19}$

\section{Benign Prostatic Tissue and ADT}

Changes in benign prostatic glands and stroma will often be the first clue that a patient has been treated with ADT. In the author's experience this is particularly the case in radical prostatectomy specimens where peripheral and transition zone compartments of the prostate can be assessed histologically. Peripheral zone changes include marked glandular atrophy with prominent basal cells and rupture of atrophic acini. Atrophic glands may rupture resulting in the presence of orphaned corpora amylacea in the prostatic stroma. Benign glands in the transition zone tend to show prominent basal cell hyperplasia (Figure 1). Squamous metaplasia may also be identified, however this was more commonly encountered when prostate cancer was treated with estrogen therapy, a treatment option that is now seldom used.

\section{Prostatic Adenocarcinoma and ADT}

Hormone-naive usual acinar-type prostatic adenocarcinoma is recognized by the classical major criteria of infiltrative architecture, nuclear atypia with prominent nucleoli, and an absence of basal cells. Prostatic adenocarcinoma showing maximum treatment effects of ADT is characterized architecturally by glands with compressed lumina or as single cells in cords, tiny clusters, or chains, or in solid sheets. The malignant cells themselves tend to have clear, vacuolated, or foamy cytoplasm with shrunken, pyknotic nuclei and inconspicuous or absent nucleoli. The pale, shrunken nature of these cells can easily lead to them being overlooked. These 'regressive changes' are usually apparent within 3 months of initiating ADT but are reversible, with nucleoli re-appearing within 20 days of cessation of ADT. ${ }^{19}$ ADT-induced changes in prostate cancer can resemble xanthomatous inflammation and can also mimic the features of the foamy gland 

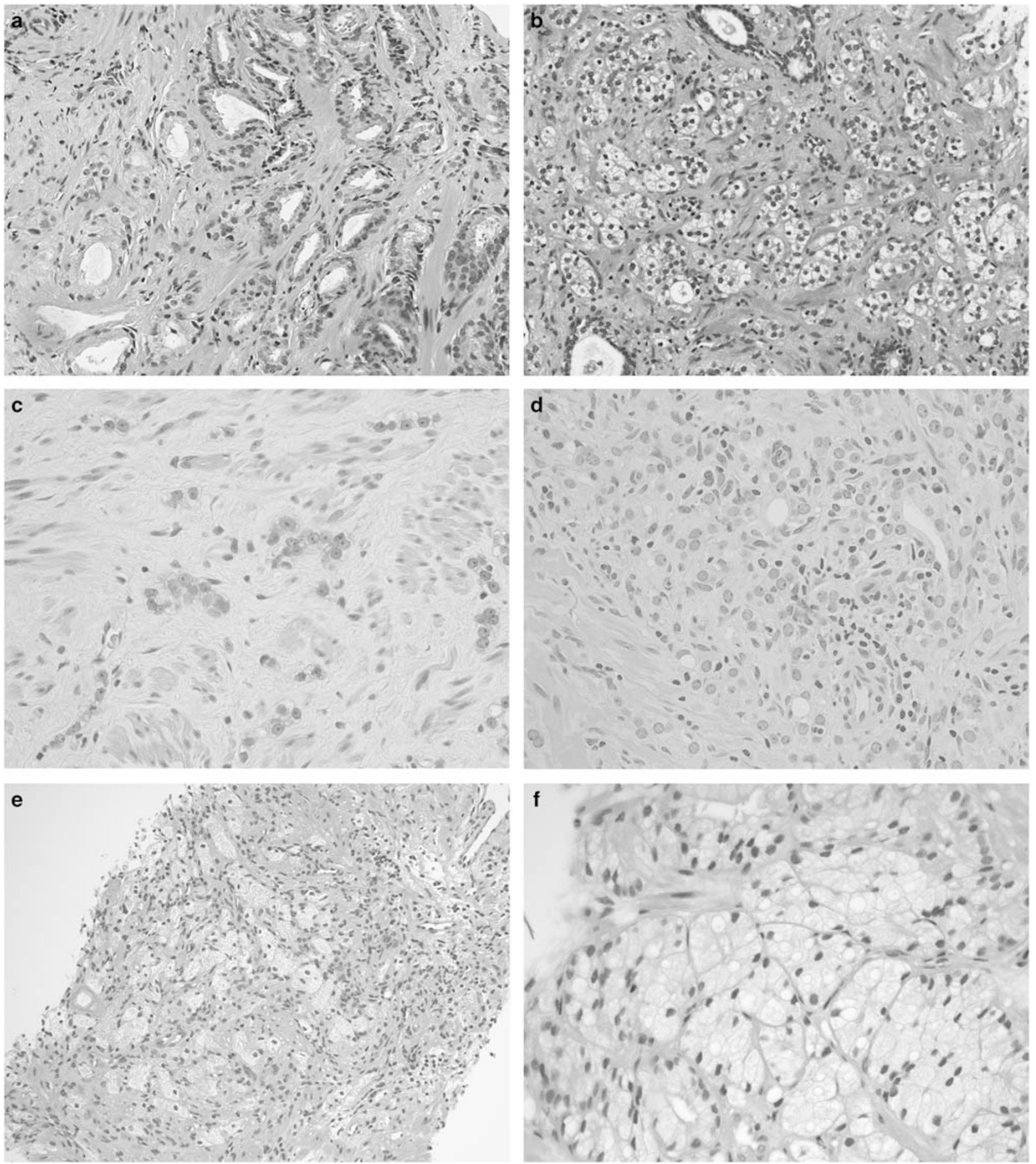

Figure 2 (a) Typical appearance of hormone therapy-naive acinar-type prostatic adenocarcinoma showing well-formed acini lined by cells with amphophilic cytoplasm and round nuclei with prominent nucleoli (hematoxylin-eosin, $\times 200$ ) compared with (b) adenocarcinoma showing maximum androgen-deprivation effects characterized by infiltrating glands with compressed lumina and cells with pale to clear cytoplasm and shrunken nuclei with indistinct or absent nucleoli (hematoxylin-eosin, $\times 200$ ). (c) Higher-magnification view of posthormone therapy adenocarcinoma illustrating single cells and cords of tumor cells within edematous stroma (hematoxylin-eosin, $\times 400$ ). (d) Higher-magnification view of post-hormone therapy adenocarcinoma illustrating tumor with an admixed mild lymphocytic infiltrate (hematoxylin-eosin, $\times 400$ ). These features exhibited by tumor showing these treatment effects can be reminiscent of the lipid-laden histiocytes comprising a prostatic xanthoma. An example of xanthoma cells is shown in e (hematoxylin-eosin, $\times 200$ ). They can also mimic the features of foamy gland variant adenocarcinoma. An example of foamy gland variant adenocarcinoma is shown in $\mathbf{f}$ (hematoxylin-eosin, $\times 400$ ). 
variant of prostatic adenocarcinoma (Figure 2). Pathologists encountering these features in prostate specimens should look for clinical information indicating previous exposure to $\mathrm{ADT}$. In particular, requisitions accompanying such biopsies can indicate markedly elevated serum PSA levels without mentioning the patient was subsequently given ADT before biopsy. The stated PSA level may not represent the actual PSA level at the time of the biopsy in such a scenario.

\section{Grading and ADT}

The architectural features of ADT-treated prostate cancer in theory meet criteria for high-grade cancer if Gleason grades were to be applied. Studies have shown that hormone-treated prostate cancer showing effects of MAB has a reduced capacity for proliferation $^{20}$ and available evidence indicates Gleason grades should not be assigned in this setting as they are not biologically relevant. ${ }^{19}$ The degree of ADT effects on tumor morphology can vary, from minimal to marked, within the same specimen. In the author's experience, this is most likely to be apparent in radical prostatectomy specimens following neoadjuvant ADT. Pathologists can include a comment describing the range of treatment effects seen. If the patient has a stated history of ADT and no treatment effects are discernable, pathologists should assign Gleason scores and include a comment stating the history of ADT is acknowledged, however treatment effects are not apparent. Clinicians should be aware of the issue of grading after ADT. The use of ADT before biopsy in patients presenting with clinically advanced disease may prevent these patients from being entered into clinical trials where Gleason scores at diagnosis are required for enrollment. The use of neoadjuvant ADT before radical prostatectomy could also be a limiting factor in terms of enrollment into clinical trials for the same reason.

The use of neoadjuvant ADT is associated with substantial reductions in tumor size in (eg, in the order of $40-60 \%$ ), along with lower pathologic stage and a lower incidence of positive resection margins compared to prostate cancers not treated with neoadjuvant ADT. In addition, no carcinoma will be found in prostatectomy specimens in upwards of $20 \%$ of cases treated in this manner. ${ }^{21}$ Despite these apparently favorable effects, the use of neoadjuvant ADT does not appear to change patient outcome. ${ }^{22}$ The study of Eftsathiou et al. ${ }^{15}$ indicates that pathologists should look for and report intraductal carcinoma (IDC) and invasive carcinoma with cribriform architecture in these cases, as these features are confirmed adverse prognostic signs in this setting.

\section{ADT and Neuroendocrine Differentiation}

The emergence of neuroendocrine (NE) differentiation following prolonged ADT is a well-recognized phenomenon in prostate cancer. ${ }^{23}$ The rapid autopsy study of Shah et $a .^{24}$ found NE differentiation in $10 \%$ of cases with castrate-resistant disease. It manifests clinically as an expanding tumor burden, often with a stable serum PSA and castrate levels of testosterone, and metastases to sites not commonly seen in usual acinar-type prostate cancer. NE differentiation can also be an incidental finding in palliative transurethral resection of prostate (TURP) specimens in patients treated with primary ADT (with or without prior or combined RT) ${ }^{25}$ Prostate cancers showing NE differentiation following ADT are typically AR-negative on immunohistochemical staining ${ }^{25}$ and are not given Gleason scores. ${ }^{26}$

\section{$5 \alpha$-Reductase Inhibitors}

5ARIs such as finasteride and dutasteride are typically used in the management of symptomatic BPH. ${ }^{27}$ These agents represent a specific and relatively mild form of ADT. The Prostate Cancer Prevention Trial (PCPT) published in 2003 looked at the potential of 5ARIs to prevent the development of prostate cancer in biopsy-negative men treated with finasteride or placebo who were followed prospectively for 3 years. While this trial reported a significant reduction in the number of biopsydetected prostate cancers in the finasteride arm, there was also a significant increase in the number of high-grade cancers found in men given this drug. ${ }^{28}$ Given the effects of ADT on prostate cancer morphology, the question of the higher Gleason scores being an artifact of 5ARI therapy was logically raised. Lucia et al. ${ }^{29}$ carried out a review of PCPT cases where the urological pathologists reviewing the cases were blinded to information concerning 5ARI therapy. These pathologists were specifically asked whether they could identify the patients treated with 5ARIs based on the presence of treatment effects attributable to ADT. The study had a negative result in that the expert pathologists could not identify any influence of 5ARIs on the morphology of prostate cancer. The increased number of high-grade cancers in the finasteride arm was not considered to be a morphology-based artifact created by 5ARI treatment and has since been attributed to improved sampling of higher-grade cancer in a gland that was reduced in size by 5ARI therapy. ${ }^{30}$ As such, Gleason scores should be provided in cancer-containing prostate specimens from men treated with 5ARIs.

\section{Radiation therapy}

Radiation therapy (RT) in prostate cancer can be used for primary treatment or adjuvant therapy following prostatectomy in men found to have locally advanced disease with positive resection margins. RT can be delivered as external beam therapy, including intensity modulated, threedimensional conformal or stereotactic delivery 

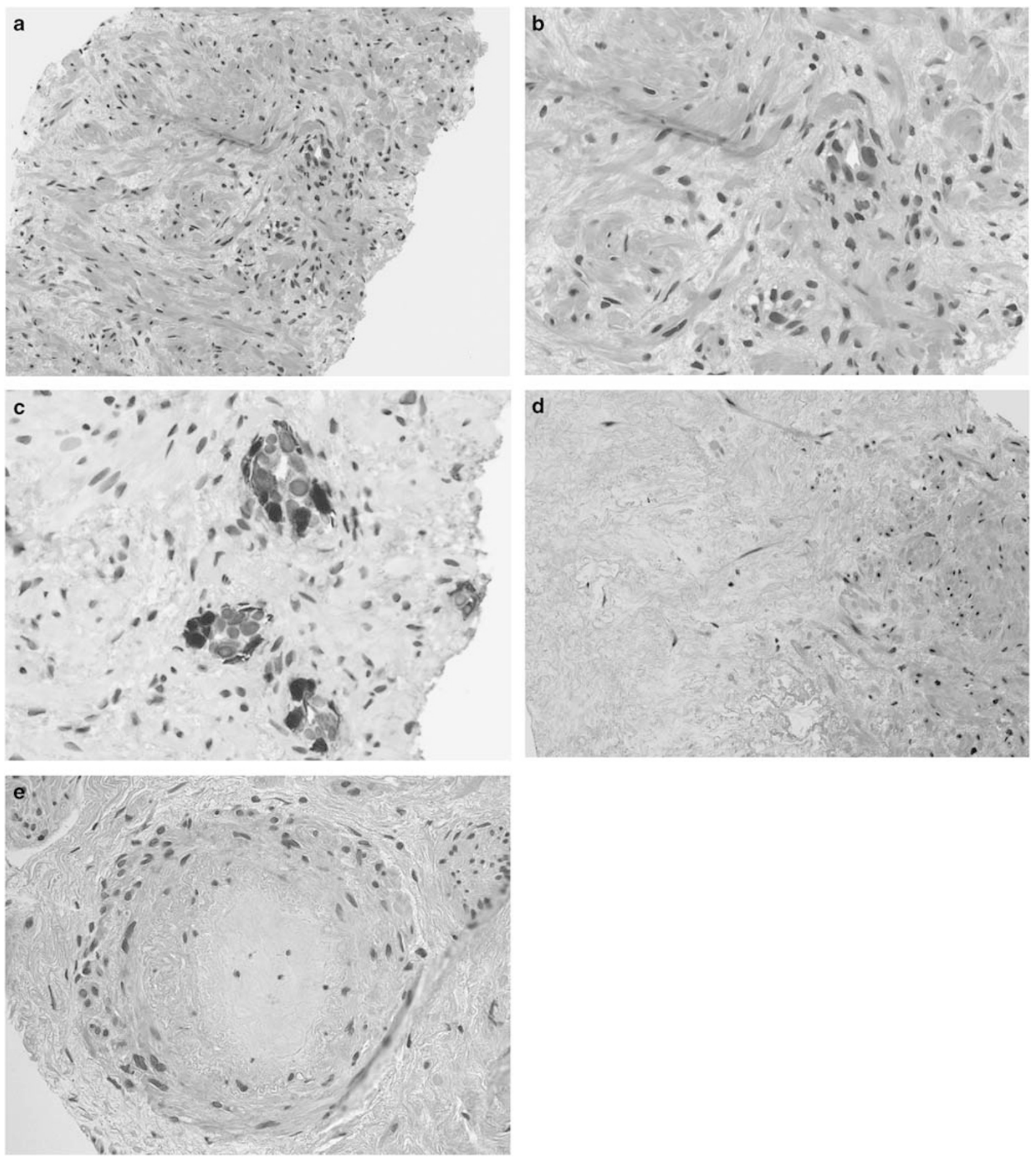

Figure 3 Appearance of benign prostate tissue after radiation therapy. (a) The overall lobular architecture of benign glands is preserved. The individual glands are atrophic and irregular in shape (hematoxylin-eosin, $\times 100$ ). (b) Higher-magnification view showing a benign gland with prominent basal cells that have hyperchromatic and irregular nuclei, often with prominent nucleoli (hematoxylin-eosin, $\times 400$ ). (c) Immunohistochemical staining with p63 and high-molecular-weight cytokeratin confirms the presence of basal cells in such acini. AMACR staining in negative (p63/HMWK/AMACR cocktail, $\times 400$ ). (d) Prostatic stroma will show variable fibrosis (hematoxylineosin, $\times 100$ ) with $(\mathbf{e})$ small caliber blood vessels often showing complete fibrous obliteration if they are recognizable (hematoxylin-eosin, $\times 200)$.

approaches or localized brachytherapy based on the implantation of radioactive seeds. External beam RT is typically given as primary therapy for locally advanced high-grade disease in patients for whom radical prostatectomy is not an option. Brachytherapy is reserved for men with low- to intermediate-risk disease (grade group 1 or 2) with no clinical or radiological evidence of extraprostatic 

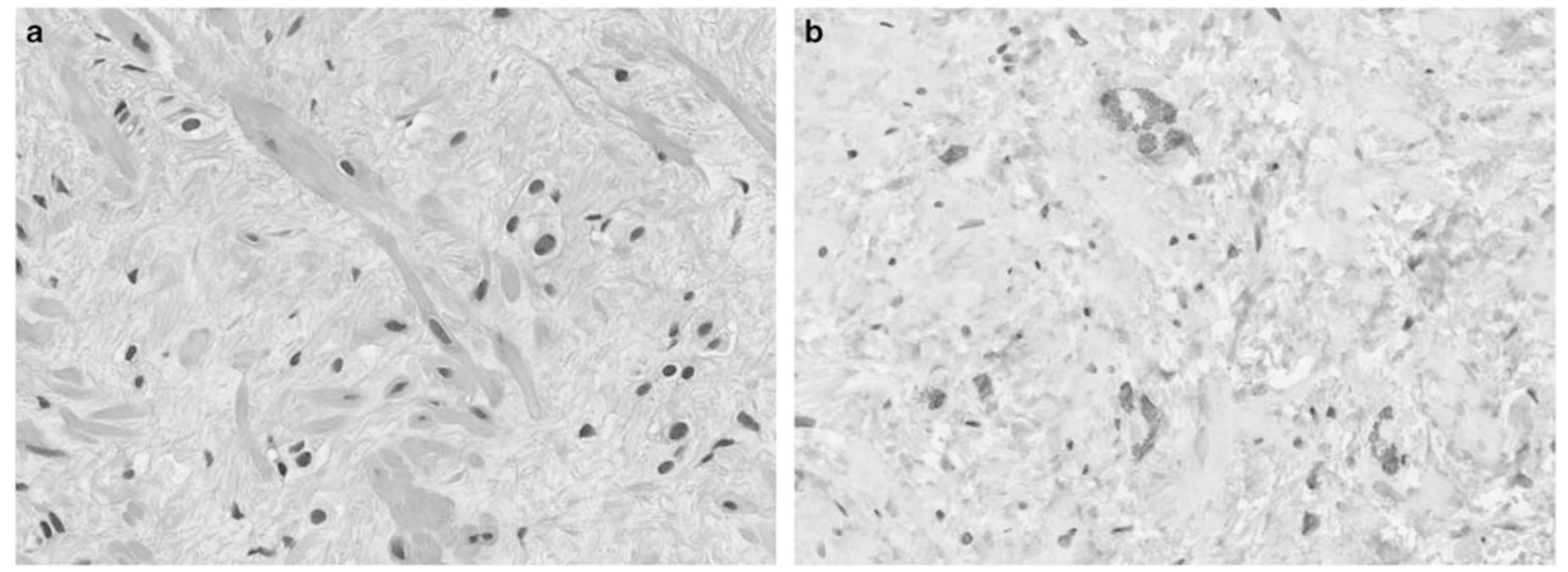

Figure 4 Prostatic adenocarcinoma showing marked radiation therapy effects characterized by (a) single cells, tiny clusters of cells, and/or small glands with a haphazard, infiltrative architecture. The malignant cells themselves have clear or pale vacuolated cytoplasm and pyknotic nuclei with smudged chromatin (hematoxylin-eosin, $\times 400$ ). On staining with p63, high-molecular-weight cytokeratin and AMACR, (b) these glands and cells show negative staining with basal cell markers and positive immunoreactivity AMACR in the majority of cases (p63/HMWK/AMACR cocktail, $\times 400)$

involvement. ${ }^{31}$ Pathologists should be aware of the fact that RT can be combined with ADT, which should be kept in mind when assessing prostate specimens from these patients. The most commonly encountered prostate specimens following RT are prostate biopsies, performed as part of routine follow-up or clinical trials. Prostate biopsies are typically obtained no sooner than 24-30 months following the completion of RT when serum PSA levels have reached their nadir and are stable. ${ }^{32,33}$ At earlier points following RT, serum PSA falls but can rebound with a temporary and benign 'PSA-bounce.' This phenomenon is typically seen 15-18 months following therapy and is not indicative of treatment failure. PSA bounces can occur following external beam or brachytherapy, occurring in $30-40 \%$ of men successfully treated with the latter modality. ${ }^{34}$ As with ADT, RT is associated with characteristic treatment-related changes in the morphology of prostate cancer as well as benign prostatic glands and stroma. These changes have been well described.11-14 The modality of RT does not appear to influence the basic morphologic changes seen in either benign or malignant prostate tissue, however the severity of treatment effects seen may differ with brachytherapy depending where tissue has been obtained in relation to the seeds. ${ }^{13}$ The three most common diagnoses for post-RT biopsies are as follows: (1) negative for malignancy, RT effects present; (2) adenocarcinoma showing RT effectsno Gleason score is assigned; and (3) adenocarcinoma showing no treatment effects with the appropriate Gleason score. Biopsy findings that do fit into one of these three categories can be reported descriptively along with an explanatory comment. $\mathrm{RT}$ induces the following changes in benign prostatic tissue; glandular atrophy with prominent basal cells usually having hyperchromatic, irregular nuclei often having prominent macronucleoli. The adjacent stroma will usually show variable fibrosis with small caliber blood vessels often showing complete fibrous obliteration if they are recognizable (Figure 3). The histologic appearance of prostate cancer following RT can vary from no discernable changes to marked changes such that it is barely recognizable histologically. Marked RT changes are characterized by single cells, clusters of cells, and/or small glands with a haphazard, infiltrative architecture. The malignant cells themselves have clear or pale vacuolated cytoplasm and pyknotic nuclei with smudged chromatin (Figure 4). The presence of perineural invasion is often helpful in the recognition of prostate cancer showing RT effects. Because of the deceptive appearance of prostate cancer following RT, ancillary staining is helpful to confirm the diagnosis of adenocarcinoma showing treatment effects. It is important to note that the performance of ancillary staining, including basal cell markers and P504S/AMACR is not changed by RT. The basal cells in benign glands show strong positivity with highmolecular-weight keratin and p63, and negative staining with P504S/AMACR (Figure 3). The malignant glands and cells will show the predicted absence of staining with basal cell markers and positive immunoreactivity with P504S/AMACR in the majority of cases (Figure 4).

\section{Gleason Grading Following RT}

The results of 24-month post-RT biopsies have been shown to predict disease-free survival. This is particularly true when treatment changes in tumor tissue are marked. Crook et al. showed that post-RT biopsies with adenocarcinoma having essentially no treatment effects had a $>55 \%$ incidence of local failure at 5 years. Negative post-RT biopsies or those described as having tumor with marked treatment effects had a 
local failure rate of $18 \%$ over the same follow-up period. ${ }^{32}$ When treatment effects are apparent, Gleason scores should not be assigned to post-RT carcinoma as they have no biological relevance. A particularly useful decision support tool to help pathologists determine whether Gleason scores should be assigned is the 1987 grading scheme of Bocking and Aufferman, ${ }^{35}$ which was subsequently modified by Crook et al. ${ }^{32}$ for the assessment of post-RT biopsies as part of a clinical trial. This scheme considers cytoplasmic and nuclear characteristics, with each being graded on a four-point (0-3) scale to give a combined score out of 6. Gleason scores should only be assigned when combined cytoplasmic and nuclear scores are either 0 (meaning no apparent treatment effect) or 1 (cytoplasmic swelling/microvesicular change or nuclear enlargement with some chromatin smudging but still visible nucleoli). Additional follow-up biopsies obtained after post-RT biopsies showing only adenocarcinoma with marked treatment effects will be negative in $30-40 \%$ of patients, providing further evidence as to the indolent nature of tumor showing treatment changes. ${ }^{32}$ The main reason for pathologists to recognize and report post-RT changes of this nature is to avoid unnecessary salvage therapy. When post-RT biopsies contain adenocarcinoma with no discernable treatment effect, the author recommends adding a comment acknowledging the history of RT and stating that no changes attributed to the RT are noted and Gleason scores can be assigned.

\section{Treatment options for clinically low-risk prostate cancer}

While the specific criteria used to define clinically low-risk prostate cancer can vary between institutions and urologists, it is generally described as having the following attributes: Gleason score $\leq 6 / 10$ (grade group 1); serum PSA value $\leq 10 \mathrm{ng} / \mathrm{ml}$; negative digital rectal exam (cT1); $\leq 30 \%$ of biopsy cores positive for cancer; and $\leq 50 \%$ involvement for the positive core containing the most cancer. ${ }^{1}$ According to some recent guidelines, Gleason score 7/10 (3+4) (grade group 2) can be considered clinically low risk if the amount of pattern 4 tumor comprises $\leq 10 \%$ of total cancer in a set of needle core biopsies. ${ }^{36}$ The basic principles of management for low-risk prostate cancer aim to balance the risk of over-treatment against the risk of missing an opportunity for cure. Current options include direct intervention by radical prostatectomy or RT, AS, and ablative therapies.

\section{Active Surveillance}

AS refers to observation with curative intent through regular monitoring of serum PSA, DRE findings, serial biopsies, and diagnostic imaging (prostate magnetic resonance imaging (MRI) in particular).
The aim is to provide active treatment when low-risk disease progresses to a higher-risk category, while avoiding the negative impacts of treating disease that remains low-risk. By definition, patients on AS will not have undergone treatment for prostate cancer and the histology of serial biopsies from these patients should not show treatment effects. Gleason scores are generally the most important determinant for whether a patient remains on AS. 1,36

\section{Ablative Therapies}

Ablative therapies introduce energy to the prostate in a minimally invasive manner to destroy cancerous foci while minimizing the damage to adjacent structures. In patients with low-risk disease, these treatments offer a potential compromise between AS and surgery or RT. They can be used to destroy onehalf of the gland (hemi-ablation) or the entire gland or to treat highly localized areas when needle biopsies and MRI information are used to map out the cancerous region. ${ }^{37}$ In addition to being used as primary therapy, ablative therapies can be used as salvage treatment in patients who have failed primary RT. ${ }^{38}$ The various options for ablative therapy include HIFU, interstitial laser ablation therapy (ILAT), vascular targeted photodynamic therapy (PDT), and cryotherapy (CT). ${ }^{3,39}$ Other ablative modalities that will not be reviewed here include irreversible electroporation and radiofrequency ablation. As a general rule, ablative treatments produce well-demarcated areas of tissue damage. With respect to cancerous areas in the prostate, these treatments either destroy cancer or leave it essentially unchanged. Changes that might impact the recognition or grading of prostate cancer are not usually seen. ${ }^{12,13,17}$

HIFU delivers intersecting ultrasound waves from a transducer either transrectally or transurethrally. The ultrasound waves are focussed on a tumor target, heating the tissue to $>60^{\circ} \mathrm{C}$ rendering the tissue nonviable. ${ }^{40}$ The operator can visually monitor the temperature of the target tissue in real time. When the optimal temperature has been reached, the transducer is moved to an adjacent field. The process is repeated until the entire region of interest has been ablated. ${ }^{39}$ While the quality of evidence for patients treated with HIFU for localized prostate cancer is very low, ${ }^{41}$ encouraging results for HIFU when used as primary therapy have been reported. In a systematic review of 20 series of patients treated with primary or salvage HIFU, Warmuth et al. ${ }^{42}$ reported overall negative biopsy rates of $86 \%$ and $80 \%$ at 3 and 15 months, respectively. HIFU does not come without adverse side effects, including erectile dysfunction and bladder outlet obstruction. ${ }^{41}$ There is still a lack of randomized trials with long-term follow-up data and there are no uniform follow-up guidelines to date. It has been the author's experience that some urologists routinely biopsy patients 
after HIFU, while others use post-HIFU PSA to determine whether follow-up biopsies are performed. The technique is user-dependent and patient selection is critical. The use of radical prostatectomy or RT as salvage therapy following failed HIFU is greatly complicated by the local tissue damage caused by this procedure. Fibrosis induced by the HIFU procedure destroys usual surgical planes resulting in increased patient morbidity ${ }^{43}$ and a distorted specimen with ragged resection margins (Figure 5). HIFU acutely induces coagulative necrosis with cystic cavitation as described in studies on canine prostates. ${ }^{44}$ Necrosis and hemorrhage was found in a small series of human radical prostatectomy specimens obtained 2 weeks after exposure to HIFU. ${ }^{45}$ Most pathologists will not encounter specimens obtained immediately after HIFU, but are more likely to receive needle biopsies 12-15 months after HIFU in patients whose post-HIFU PSA is rising. Biopsies from successfully treated areas most commonly show dense stromal fibrosis and hemosiderin deposition after a mean follow-up period of 15.3 months. ${ }^{17}$ The fibrosis becomes more extensive as the duration of follow-up increases. The interface between treated and untreated areas is usually welldemarcated and readily apparent at scanning magnification. Biopsies obtained after shorter follow-up intervals of $\sim 8$ months will show coagulative necrosis in $10-15 \%$ of patients. Post-HIFU biopsies performed because of rising PSA levels will show adenocarcinoma in upwards of $77 \%$ of cases. The adenocarcinoma in such biopsies shows no obvious treatment-induced changes and Gleason scores can reliably be assigned. Likewise, the performance of ancillary immunohistochemical staining such as p63, 34 $\beta$ E12, and AMACR are unaffected by HIFU therapy. ${ }^{17}$ Rarely, a short course of cytoreductive ADT may be given prior to HIFU. ${ }^{18}$ Adenocarcinoma and benign tissue in biopsies obtained 3-4 months after the ADT and HIFU may show the expected morphologic changes associated with ADT.

In ILAT, $830 \mathrm{~nm}$ diode laser probes are inserted into the prostate through a transperineal route under MRI guidance. MRI is also used to monitor temperature changes in the target tissue in real time. ${ }^{2}$ Radical prostatectomy specimens obtained $\sim 1$ week after ILAT therapy show well-defined areas of necrosis with a surrounding rim of hemorrhagic tissue. ${ }^{2}$ Needle biopsies obtained $\sim 6$ months after ILAT will show the same basic changes. ${ }^{12,13}$ The treated area is usually sharply demarcated from the untreated prostate. At higher magnification, ghosts of malignant acini are identifiable within the areas of coagulative necrosis. The intervening rim of hemorrhagic tissue between the coagulative necrosis and untreated tissue is also usually appreciable in postILAT biopsies. In selected patients with MRI-visible prostate cancers ILAT appears to be associated with encouraging short-term outcome and morbidity profiles, ${ }^{46}$ however longer-term follow-up data is lacking (Figure 6).

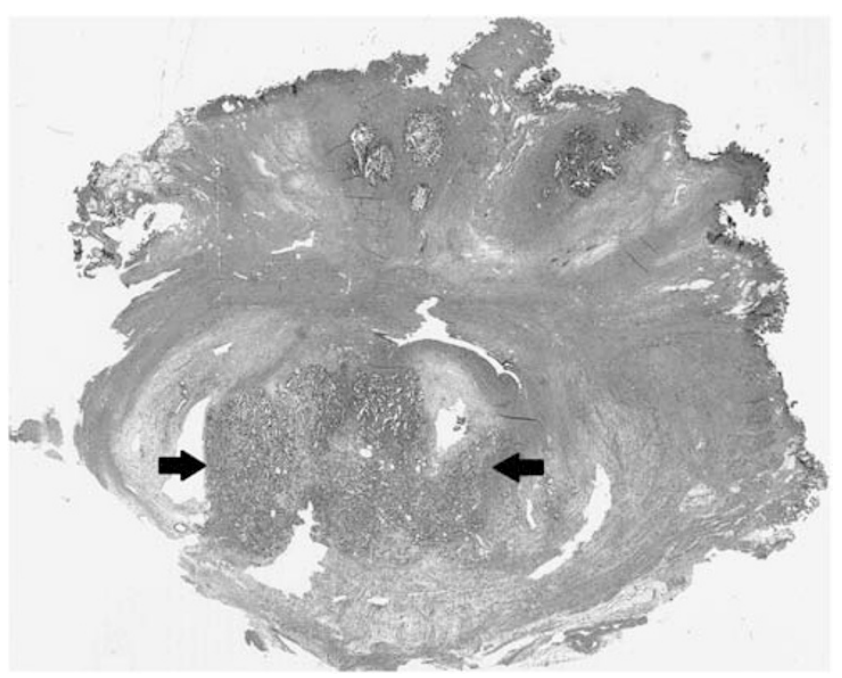

Figure 5 Wholemount hematoxylin-eosin section from a salvage radical prostatectomy specimen in a patient who underwent highintensity focussed ultrasound therapy 17 months earlier. The patient had a serum PSA of $2.03 \mathrm{ng} / \mathrm{ml}$ and biopsy-proven persistent adenocarcinoma. Note the ragged peripheral resection margin around the entire gland and seminal vesicles characterized by marked fibrosis attributable to the ablative therapy. The viable adenocarcinoma (arrows) is located in the left anterior aspect of the prostate.

In vascular targeted PDT, biologically inactive photosensitizing agents derived from bacteriochlorophylls are injected intravenously. These compounds become activated by visible light in the spectrum of $732-763 \mathrm{~nm}$, which is delivered by optical fibers placed directly in the target tissue. This causes the formation of reactive oxygen species, which in turn causes thrombosis in the vascular bed of the targeted area with the subsequent development of localized necrosis. ${ }^{39}$ The selectivity and extent of treatment-induced tissue damage is determined by the dose of the photosensitizer and the number and location of optical fibers used. It is possible to destroy the entire prostate by this method. PDT was initially investigated in clinical trials as salvage therapy in men who failed primary RT. ${ }^{47,48}$ Post-PDT biopsies in such patients obtained 6 months after treatment show well-demarcated areas of dense fibrosis. ${ }^{12,13,48}$ As with other ablative therapies, areas of viable adenocarcinoma outside of the treated area show no obvious treatment-induced morphology changes in post-PDT biopsies. Recent clinical trial results assessing the use of PDT in the primary treatment of low-risk prostate cancer are encouraging. Azzouzi et al. ${ }^{49}$ randomized men with low-risk prostate cancer in 47 European centers who had not received any prior therapy to either AS or PDT. At 24 months of follow-up, men who received padeliporfin-based PDT showed disease progression in $28 \%$ of cases (58 of 206 patients) as compared to $58 \%$ of men (120 of 207 patients) in the AS arm. Negative biopsies were found in $49 \%$ of men in the PDT group as compared to $14 \%$ of men in the AS group. In addition to apparent benefits in terms of 

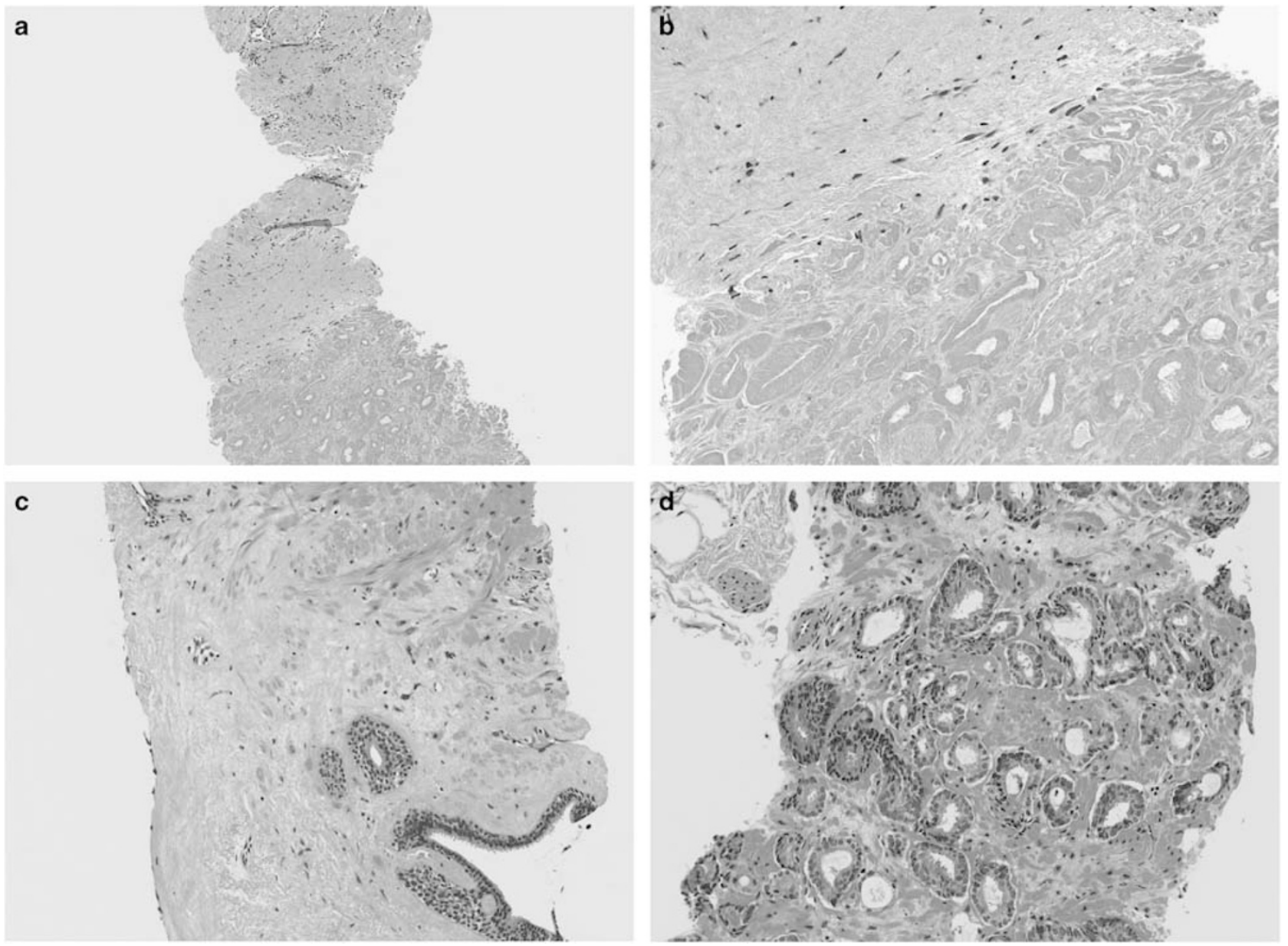

Figure 6 Appearance of prostate tissue in a needle biopsy obtained 9 months after attempted complete gland ablation by high-intensity focussed ultrasound (HIFU) therapy. (a) The interface between treated and untreated areas is usually well-demarcated and readily apparent at scanning magnification (hematoxylin-eosin, $\times 50$ ). (b) Post-HIFU biopsies obtained in this time period often show coagulative necrosis in the treated area as indicated by the 'ghosts' of nonviable malignant glands (hematoxylin-eosin, $\times 200$ ). (c) Benign glands surrounded by fibrotic stroma at the boundary of a treated area (hematoxylin-eosin, $\times 100$ ). (d) Viable adenocarcinoma in unsuccessfully treated areas shows no obvious treatment-induced changes and reliable Gleason scores can be assigned (hematoxylin-eosin, $\times 100$ ). The features described above generally apply to all ablative therapies, including interstitial laser ablation, photodynamic therapy, and cryotherapy.

local cancer control, PDT therapy was well-tolerated in this cohort.

In contrast to HIFU and ILAT, which cause tissue destruction by delivering heat to the prostate, cryotherapy is based on cooling the target tissue to $-40^{\circ} \mathrm{C}$. Needles are introduced into the prostate via the perineum through which cold gases are delivered directly into the target tissue, which induces the formation of an ice ball. Tissue destruction occurs when the ice ball thaws. ${ }^{3}$ As with other forms of ablative therapy, fibrosis, coagulative necrosis, and hemosiderin deposition are characteristic findings in post-cryotherapy biopsies.

\section{Chemotherapy and immune therapy}

Contemporary and emerging options for androgenindependent, systemically advanced prostate cancer include chemotherapy and immune-based approaches. These approaches have historically been used as palliative measures. Most pathologists would not encounter diagnostic specimens from these patients aside from autopsy, palliative TURP procedures, or biopsies performed as part of clinical trials. As such, there are few published descriptions of treatment-induced changes in the morphology of prostate cancer in this setting. A complicating issue with respect to assessing treatment-induced changes specific to these agents in androgenindependent disease, is the fact that most if not all of these patients will have been treated with ADT and/or RT and possibly salvage ablative therapy. Nonetheless, as new clinical trials for hormonesensitive and -resistant prostate cancer appear, pathologists will be required to review biopsies to document treatment-related changes that could 
signal hope to patients with an otherwise dismal prognosis.

Chemotherapy options for castrate-resistant prostate cancer include cabiztaxel, docetaxel, mitoxantrone, and estramustine. ${ }^{50}$ Changes specific to these agents are best described in clinical trials involving neoadjuvant treatment before prostatectomy in the absence of the confounding influence of prior ADT or RT. O'Brien et $a l .{ }^{51}$ described treatment-induced histopathologic changes in 50 high-risk prostate cancer patients treated with pre-prostatectomy docetaxel and mitoxantrone. These authors assessed the prognostic significance of several histopathologic features over a median follow-up period of 65 months during which the overall relapse-free survival rates were $65 \%$ and $49 \%$ at 2 and 5 years, respectively. Pre-treatment biopsies were available for comparison from 22 of these patients. The most common features in post-treatment prostatectomy specimens included collapsed inconspicuous glands lacking visible lumina (46\% of cases), small inconspicuous tumor cells with pyknotic, basophilic nuclei, scant cytoplasm (28\%), and prominent cytoplasmic vacuolization in tumor cells (26\%). IDC and cribriform architecture were identified in $20 \%$ and $14 \%$ of cases, respectively. One case featured large pleomorphic nuclei with abundant eosinophilic cytoplasm. Cribriform architecture, inconspicuous collapsed malignant glands, and vacuolated tumor cell cytoplasm were noted in 3 of the 22 pretreatment biopsy specimens available for review. While possibly attributable sampling issues, it was interesting to note that IDC was not found in pretreatment biopsies. Both IDC and cribriform architecture were associated with shorter recurrence-free survival in univariate and multivariate analyses. IDC alone was significantly correlated with the presence of lymph node metastases in multivariable logistic regression analyses when lymph node status was set as the dependent variable.

Immune therapy uses the patient's own immune system to eradicate tumor cells. ${ }^{9,52,53}$ Current and evolving immune therapy approaches include cancer vaccines, which target the cancer with educated $\mathrm{T}$ cells through endogenous $\mathrm{T}$-cell receptors (ie, sipuleucel-T), immune checkpoint inhibitors, which remove signals blocking the activation of $\mathrm{T}$ cells, which would otherwise target and eliminate tumor cells (ie, CTLA-4, PD-1, PD-L1 inhibitors, with imilipumab as one example) and adoptive cellular therapy (ie, chimeric antigen receptor $\mathrm{T}$ cells). Morphologic changes induced in benign and malignant prostate tissue induced by immune therapy have yet to be described. As with standard chemotherapy, it may be difficult to isolate changes specific to any of these agents in situations where patients have previously been treated with ADT and/ or RT.

\section{Nutritional and herbal supplements}

Vitamins D and E, soy, selenium, green tea, and saw palmetto berry extract have been suggested to have a role in the prevention of prostate cancer or as agents to promote general prostate health. ${ }^{54}$ No specific morphologic changes have reported to date. It appears unlikely these agents have any impact on the ability of pathologists to recognize or grade prostatic adenocarcinoma in specimens obtained from patients using one or more these supplements.

\section{Conclusions}

Accurate interpretation and reporting of posttreatment prostate specimens by pathologists requires a working knowledge of the various nonsurgical treatment options and the effects of each on both benign and malignant prostate tissue. This is particularly true when specimens are received without clinical information indicating prior nonsurgical therapy. In the absence of supplied history, pathologists encountering the features suggestive of prior nonsurgical treatment are encouraged to review patient records and/or communicate directly with clinical colleagues to determine what treatment was given and when.

\section{Disclosure/conflict of interest}

The author declares no conflict of interest.

\section{References}

1 Chen RC, Rumble RB, Loblaw DA, et al. Active surveillance for the management of localized prostate cancer (Cancer Care Ontario Guideline): American Society of Clinical Oncology Clinical Practice Guideline Endorsement. J Clin Oncol 2016;34:2182-2190.

2 Lindner U, Lawrentschuk N, Weersink RA, et al. Focal laser ablation for prostate cancer followed by radical prostatectomy: validation of focal therapy and imaging accuracy. Eur Urol 2010;57:1111-1114.

3 Perera M, Krishnananthan N, Lindner U, et al. An update on focal therapy for prostate cancer. Nat Rev Urol 2016;13:641-653.

4 Mottet N, Bellmunt J, Bolla M, et al. EAU-ESTRO-SIOG Guidelines on Prostate Cancer. Part 1: Screening, Diagnosis, and Local Treatment with Curative Intent. Eur Urol 2017;71:618-629.

5 Gravis G, Audenet F, Irani J, et al. Chemotherapy in hormone-sensitive metastatic prostate cancer: evidences and uncertainties from the literature. Cancer Treat Rev 2017;55:211-217.

6 Lorente D, Fizazi K, Sweeney C, et al. Optimal treatment sequence for metastatic castration-resistant prostate cancer. Eur Urol Focus 2016;2:488-498.

7 Cornford P, Bellmunt J, Bolla M, et al. EAU-ESTROSIOG Guidelines on Prostate Cancer. Part II: Treatment of Relapsing, Metastatic, and Castration-Resistant Prostate Cancer. Eur Urol 2017;71:630-642. 
8 Golabek T, Belsey J, Drewa T, et al. Evidence-based recommendations on androgen deprivation therapy for localized and advanced prostate cancer. Cent European J Urol 2016;69:131-138.

9 Yeku O, Slovin SF. Immune therapy for prostate cancer. Cancer J 2016;22:334-341.

10 Francini E, Taplin ME. Prostate cancer: developing novel approaches to castration-sensitive disease. Cancer 2017;123:29-42.

11 Bostwick DG, Meiers I. Diagnosis of prostatic carcinoma after therapy. Arch Pathol Lab Med 2007;131: 360-371.

12 Evans AJ, Ryan P, Van derKwast T. Treatment effects in the prostate including those associated with traditional and emerging therapies. Adv Anat Pathol 2011;18: 281-293.

13 Srigley JR, Delahunt B, Evans AJ. Therapy-associated effects in the prostate gland. Histopathology 2012;60: 153-165.

14 Mazzucchelli R, Scarpelli M, Lopez-Beltran A, et al. Treatment effects in prostate cancer following traditional and emerging therapies. Int J Immunopathol Pharmacol 2013;26:291-298.

15 Efstathiou E, Abrahams NA, Tibbs RF, et al. Morphologic characterization of preoperatively treated prostate cancer: toward a post-therapy histologic classification. Eur Urol 2010;57:1030-1038.

16 Brown JA, Garlitz C, Strup SE, et al. Laparoscopic radical prostatectomy after neoadjuvant hormonal therapy: an apparently safe and effective procedure. J Laparoendosc Adv Surg Tech A 2004;14:335-338.

17 Ryan P, Finelli A, Lawrentschuk N, et al. Prostatic needle biopsies following primary high intensity focused ultrasound (HIFU) therapy for prostatic adenocarcinoma: histopathological features in tumour and non-tumour tissue. J Clin Pathol 2012;65:729-734.

18 Elterman DS, Barkin J, Radomski SB, et al. Results of high intensity focused ultrasound treatment of prostate cancer: early Canadian experience at a single center. Can J Urol 2011;18:6037-6042.

19 Tetu B. Morphological changes induced by androgen blockade in normal prostate and prostatic carcinoma. Best Pract Res Clin Endocrinol Metab 2008;22:271-283.

20 van der Kwast TH, Tetu B, Candas B, et al. Prolonged neoadjuvant combined androgen blockade leads to a further reduction of prostatic tumor volume: three versus six months of endocrine therapy. Urology 1999;53:523-529.

21 Kollermann J, Caprano J, Budde A, et al. Follow-up of nondetectable prostate carcinoma (pT0) after prolonged PSA-monitored neoadjuvant hormonal therapy followed by radical prostatectomy. Urology 2003;62: 476-480.

22 Yee DS, Lowrance WT, Eastham JA, et al. Long-term follow-up of 3-month neoadjuvant hormone therapy before radical prostatectomy in a randomized trial. BJU Int 2010;105:185-190.

23 Alberti C. Neuroendocrine differentiation in prostate carcinoma: focusing on its pathophysiologic mechanisms and pathological features. G Chir 2010;31: 568-574.

24 Shah RB, Mehra R, Chinnaiyan AM, et al. Androgenindependent prostate cancer is a heterogeneous group of diseases: lessons from a rapid autopsy program. Cancer Res 2004;64:9209-9216.

25 Evans AJ, Humphrey PA, Belani J, et al. Large cell neuroendocrine carcinoma of prostate: a clinicopathologic summary of 7 cases of a rare manifestation of advanced prostate cancer. Am J Surg Pathol 2006;30:684-693.

26 Fine SW. Variants and unusual patterns of prostate cancer: clinicopathologic and differential diagnostic considerations. Adv Anat Pathol 2012;19:204-216.

27 Oelke M, Bachmann A, Descazeaud A, et al. EAU guidelines on the treatment and follow-up of nonneurogenic male lower urinary tract symptoms including benign prostatic obstruction. Eur Urol 2013;64: $118-140$.

28 Thompson IM, Goodman PJ, Tangen CM, et al. The influence of finasteride on the development of prostate cancer. N Engl J Med 2003;349:215-224.

29 Lucia MS, Epstein JI, Goodman PJ, et al. Finasteride and high-grade prostate cancer in the Prostate Cancer Prevention Trial. J Natl Cancer Inst 2007;99:1375-1383.

30 Serfling R, Shulman M, Thompson GL, et al. Quantifying the impact of prostate volumes, number of biopsy cores and 5alpha-reductase inhibitor therapy on the probability of prostate cancer detection using mathematical modeling. J Urol 2007;177:2352-2356.

31 Zaorsky NG, Davis BJ, Nguyen PL, et al. The evolution of brachytherapy for prostate cancer. Nat Rev Urol 2017;14:415-439.

32 Crook JM, Malone S, Perry G, et al. Twenty-four-month postradiation prostate biopsies are strongly predictive of 7-year disease-free survival: results from a Canadian randomized trial. Cancer 2009;115:673-679.

33 Crook JM, Bahadur YA, Bociek RG, et al. Radiotherapy for localized prostate carcinoma. The correlation of pretreatment prostate specific antigen and nadir prostate specific antigen with outcome as assessed by systematic biopsy and serum prostate specific antigen. Cancer 1997;79:328-336.

34 Naghavi AO, Strom TJ, Nethers K, et al. Clinical implications of a prostate-specific antigen bounce after radiation therapy for prostate cancer. Int J Clin Oncol 2015;20:598-604.

35 Bocking A, Auffermann W. Cytological grading of therapy-induced tumor regression in prostatic carcinoma: proposal of a new system. Diagn Cytopathol 1987;3:108-111.

36 Morash C, Tey R, Agbassi C, et al. Active surveillance for the management of localized prostate cancer: Guideline recommendations. Can Urol Assoc J 2015;9: 171-178.

37 Woodrum DA, Kawashima A, Gorny KR, et al. Prostate cancer: state of the art imaging and focal treatment. Clin Radiol 2017;72:665-679.

38 van den Bos W, Muller BG, de Bruin DM, et al. Salvage ablative therapy in prostate cancer: international multidisciplinary consensus on trial design. Urol Oncol 2015;33:495 e1-495 e7.

39 Lindner U, Trachtenberg J, Lawrentschuk N. Focal therapy in prostate cancer: modalities, findings and future considerations. Nat Rev Urol 2010;7:562-571.

40 ter Haar GR. High intensity focused ultrasound for the treatment of tumors. Echocardiography 2001;18:317-322.

41 Veereman G, Jonckheer P, Desomer A, et al. Systematic review of the efficacy and safety of high-intensity focussed ultrasound for localised prostate cancer. Eur Urol Focus 2015;1:158-170.

42 Warmuth M, Johansson T, Mad P. Systematic review of the efficacy and safety of high-intensity focussed ultrasound for the primary and salvage treatment of prostate cancer. Eur Urol 2010;58:803-815. 
43 Lawrentschuk N, Finelli A, Van der Kwast TH, et al. Salvage radical prostatectomy following primary high intensity focused ultrasound for treatment of prostate cancer. J Urol 2011;185:862-868.

44 Kincaide LF, Sanghvi NT, Cummings O, et al. Noninvasive ultrasonic subtotal ablation of the prostate in dogs. Am J Vet Res 1996;57:1225-1227.

45 Van Leenders GJ, Beerlage HP, Ruijter ET, et al. Histopathological changes associated with high intensity focused ultrasound (HIFU) treatment for localised adenocarcinoma of the prostate. J Clin Pathol 2000;53: 391-394.

46 Eggener SE, Yousuf A, Watson S, et al. Phase II evaluation of magnetic resonance imaging guided focal laser ablation of prostate cancer. J Urol 2016;196: 1670-1675.

47 Trachtenberg J, Bogaards A, Weersink RA, et al. Vascular targeted photodynamic therapy with palladium-bacteriopheophorbide photosensitizer for recurrent prostate cancer following definitive radiation therapy: assessment of safety and treatment response. J Urol 2007;178:1974-1979 discussion 9.

48 Trachtenberg J, Weersink RA, Davidson SR, et al. Vascular-targeted photodynamic therapy (padoporfin, WST09) for recurrent prostate cancer after failure of external beam radiotherapy: a study of escalating light doses. BJU Int 2008;102:556-562.

49 Azzouzi AR, Vincendeau S, Barret E, et al. Padeliporfin vascular-targeted photodynamic therapy versus active surveillance in men with low-risk prostate cancer (CLIN1001 PCM301): an open-label, phase 3, randomised controlled trial. Lancet Oncol 2017;18:181-191.

50 Mizokami A, Kadono Y, Kitagawa Y, et al. Therapies for castration-resistant prostate cancer in a new era: The indication of vintage hormonal therapy, chemotherapy and the new medicines. Int J Urol 2017;24:566-572.

51 O'Brien C, True LD, Higano CS, et al. Histologic changes associated with neoadjuvant chemotherapy are predictive of nodal metastases in patients with high-risk prostate cancer. Am J Clin Pathol 2010;133: 654-661.

52 Rijnders M, de Wit R, Boormans JL, et al. Systematic review of immune checkpoint inhibition in urological cancers. Eur Urol 2017;72:411-423.

53 Madan RA, Gulley JL. Prostate cancer immunotherapy: the path forward. Curr Opin Support Palliat Care 2017;11:225-230.

54 Yacoubian A, Dargham RA, Khauli RB, et al. Overview of dietary supplements in prostate cancer. Curr Urol Rep 2016;17:78. 